\title{
A spectral element method calculation of extraordinary light transmission through periodic subwavelength slits
}

\author{
Ma Luo, ${ }^{1}$ Qing Huo Liu, ${ }^{1, *}$ and Junpeng Guo ${ }^{2}$ \\ ${ }^{1}$ Department of Electrical and Computer Engineering, Duke University, Durham, North Carolina 27708, USA \\ ${ }^{2}$ Department of Electrical and Computer Engineering, University of Alabama in Huntsville, Huntsville, Alabama \\ 35899, USA \\ *Corresponding author: qhliu@ee.duke.edu
}

Received October 20, 2009; accepted November 30, 2009;

posted January 13, 2010 (Doc. ID 118795); published February 25, 2010

\begin{abstract}
A spectral element method together with a surface integral equation as the radiation boundary condition is used to simulate the scattering properties of periodic subwavelength slits. The surface integral equation utilizes the periodic Green's function in the wave number space and is solved by the method of moments, while the interior inhomogeneous medium is modeled by the spectral element method. The solution convergence is found to be exponential; i.e., the error decreases exponentially with the order of basis functions. To our knowledge, such a fast solver with spectral accuracy is new in the scattering problem of periodic structures. Scattering properties of a gold slit grid within the whole wavelength-incidence angle parameter space are investigated, with the confirmation that strong transmission of light through subwavelength slits is achievable.

(C) 2010 Optical Society of America
\end{abstract}

OCIS codes: $000.4430,160.5298$.

\section{INTRODUCTION}

Periodic structures such as photonic crystals (PCs) have many applications. Among different periodic structures, in this work we are particularly interested in twodimensional (2D) structures with one-dimensional (1D) periodicity as shown in Fig. 1 , where a $1 \mathrm{D}$ periodic $\mathrm{PC}$ along the $x$ direction separates two half spaces of different materials. The incident light from the upper half space can undergo an interesting scattering process by the $1 \mathrm{D}$ $\mathrm{PC}$, especially when the periodic structure consists of dispersive media (such as a metal in the optical frequency regime). An analysis of such structures requires a very accurate modeling technique in order to capture narrowband phenomena such as the extraordinary light transmission through subwavelength slits.

We propose the combination of the spectral element method (SEM) with a surface integral equation to solve this problem with high accuracy. The SEM [1-3] is a special kind of finite element method whose basis functions are constructed by high-order Gauss-Lobatto-Legendre (GLL) polynomials. This method was shown to have exponential convergence versus the order of basis functions when it is used to solve the band structure of $2 \mathrm{D}$ periodic PCs [4]. When the 2D PCs have a finite size along one $(y)$ direction and an infinite size along another $(x)$ direction, the scattering properties become an important issue. At the boundary perpendicular to the periodic direction with an infinite size, the periodic boundary condition is still applicable. At the other two boundaries that separate the upper and lower half spaces, a surface integral equation [5] is used to describe the radiation boundary condition with a periodic Green's function. Previously, the spectral integral method (SIM) [6,7] has been used to solve the surface integral equation with spectral accuracy. The key step is to remove the singular point of the Green's function when the observation point coincides with the source point by subtracting a function with the same singularity. The analytical form of the Fourier transform of this function is known, which is used to calculate the surface integral of this function. In this paper, we propose an alternative scheme to treat the singularity of the periodic Green's function. Here, the periodic Green's function is calculated in the wave number domain, and transferred back to the real space, which turns out to be a superposition of a set of plane waves. We use this wave number representation of the periodic Green's function to solve the surface integral equation so that the accuracy and efficiency of the method of moments (MoM) can be as good as the SEM. Therefore, the whole simulation method can have an exponential convergence.

This 2D hybrid method is a general solver for $1 \mathrm{D}$ periodic structures separating two different half spaces. As one special application, it can be used to solve the problem of scattering properties of a slit grid. One of the most interesting topics in this area is the scattering of a slit grid consisting of a dispersive metal. The incident light can excite surface plasmon polaritons (SPPs) [8], which are coupled electromagnetic modes between free electron density oscillations and electromagnetic fields. Since there is a momentum mismatch between the SPP and the incident photon, it needs additional momentum to excite the SPP. Many schemes have been proposed to provide the additional momentum, such as Kretschmann and Raether [9], Otto [10], and grating [11,12] schemes. The re- 


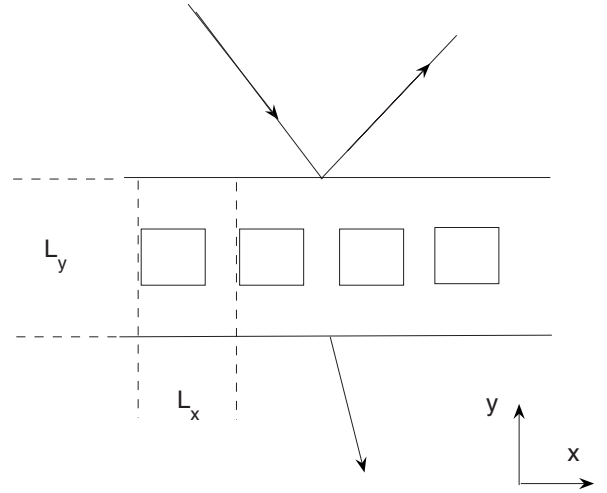

Fig. 1. Geometry of the $2 \mathrm{D}$ scattering problem with $1 \mathrm{D}$ periodicity.

search on the exciting SPP attracts wide attention because it is very useful for applications in biochemical sensors [13-15]. In this paper, we use the SEM with the MoM to calculate the transmission and absorption rates of a 1D periodic gold slit grid in the whole wavelengthincidence angle parameter space. The parameter region of the extraordinary transmission and absorption in the parameter space is investigated.

\section{FORMULATION}

The configuration of the problem under consideration is shown in Fig. 1, which is a $2 \mathrm{D}$ scattering problem with a 1D periodicity along the $\hat{x}$ direction. We investigate this $2 \mathrm{D}$ scattering problem in the frequency domain by solving the source-free Helmholtz equation of the electric field for the transverse-magnetic (TM) mode

$$
\nabla \times\left[\mu_{r s}^{-1}\left(\nabla \times E_{z} \hat{z}\right)\right]-k_{0}^{2} \varepsilon_{r z} E_{z} \hat{z}=0,
$$

or the Helmholtz equation of the magnetic field for the transverse-electric (TE) mode

$$
\nabla \times\left[\varepsilon_{r s}^{-1}\left(\nabla \times H_{z} \hat{z}\right)\right]-k_{0}^{2} \mu_{r z} H_{z} \hat{z}=0,
$$

where $k_{0}=\omega / c$ is the wave number in vacuum with $\omega$ being the circular frequency and $c$ being the speed of light in vacuum. The materials are assumed to be in-plane anisotropic, so the relative permittivity and permeability tensors are

$$
\mu_{r}=\left[\begin{array}{cc}
\mu_{r s} & 0 \\
0 & \mu_{r z}
\end{array}\right], \quad \varepsilon_{r}=\left[\begin{array}{cc}
\varepsilon_{r s} & 0 \\
0 & \varepsilon_{r z}
\end{array}\right]
$$

where

$$
\mu_{r s}=\left[\begin{array}{cc}
\mu_{x x} & \mu_{x y} \\
\mu_{y x} & \mu_{y y}
\end{array}\right] \quad \mu_{r z}=\mu_{z z}, \quad \varepsilon_{r s}=\left[\begin{array}{cc}
\varepsilon_{x x} & \varepsilon_{x y} \\
\varepsilon_{y x} & \varepsilon_{y y}
\end{array}\right]
$$

and $\varepsilon_{r z}=\varepsilon_{z z}$. Because of the duality of the electromagnetic problem, the formulas for the TM mode are made the same as the formulas for the TE mode by changing the magnetic field into an electric field and exchanging permittivity and permeability. As a result, we only discuss the formulas for the TE mode. Another reason why we focus on the TE mode is that the SPP only exists in the TE mode. The computation domain is an $L_{x} \times L_{y}$ rectangle with $L_{x}\left(L_{y}\right)$ being the length along the $\hat{x}(\hat{y})$ direction. The system is periodic along the $\hat{x}$ direction with period $L_{x}$, so that the real physical problem is a layer along the $\hat{x}$ direction with the periodic structure inside the layer, as shown in Fig. 1.

Following the process of the finite element method, the weak form of Eq. (2) is

$$
\begin{gathered}
\int_{\Gamma} d \mathbf{r}\left\{\left(\nabla \times W_{j}^{*} \hat{z}\right) \cdot\left[\varepsilon_{r s}^{-1}\left(\nabla \times H_{z} \hat{z}\right)\right]-k_{0}^{2} \mu_{r z} W_{j}^{*} H_{z}\right\} \\
\quad-j k_{0} \sum_{b=1,2} \int_{\partial \Gamma_{b}} d x W_{j}^{*} M_{z}=0,
\end{gathered}
$$

where $M_{z}$ is the magnetic current density defined as $M_{z}$ $=-\sqrt{\varepsilon_{0} / \mu_{0}} \hat{t} \cdot \mathbf{E}$, with $\hat{t}$ being the tangential unit vector. In the above, $W_{j}$ is the $j$ th test function, $\Gamma$ is the rectangular region, * denotes the complex conjugate, and $\partial \Gamma_{1}$ and $\partial \Gamma_{2}$ are the upper and lower boundaries of the rectangular region, respectively. The boundary integrations of the left and right boundaries are canceled out because of the periodic boundary condition. The magnetic field and the magnetic current density at the upper and lower boundaries are expanded by a set of basis functions, which is the same set as the test functions. The basis functions are constructed from the GLL polynomials in the reference domain and mapped into the real space elements given by meshing of the system [4]. Each basis function has an associated nodal point and the expansion coefficients of the basis functions are the value of the magnetic field or magnetic current at the nodal points. After taking the expansion coefficients of the basis functions out of the integration, the weak-form equation is converted into a matrix equation

$$
\mathbf{S}\left[\begin{array}{c}
\mathbf{H}^{i} \\
\mathbf{H}^{b 1} \\
\mathbf{H}^{b 2}
\end{array}\right]+\mathbf{T}^{(1)} \mathbf{M}^{b 1}+\mathbf{T}^{(2)} \mathbf{M}^{b 1}=0
$$

where $\mathbf{H}^{i}$ is the unknown magnetic field vector at the nodal points in the interior region of $\Gamma, \mathbf{H}^{b 1}$ and $\mathbf{H}^{b 2}$ are the unknown magnetic field vectors at the nodal points on the upper and lower boundaries, and $\mathbf{M}^{b 1}$ and $\mathbf{M}^{b 2}$ are the unknown magnetic current density vectors at the nodal points on the upper and lower boundaries, respectively. The matrix elements of $\mathbf{S}$ and $\mathbf{T}$ are defined as

$$
S_{j, k}=\int_{\Gamma} d \mathbf{r}\left\{\left(\nabla \times W_{j}^{*} \hat{z}\right) \cdot\left[\varepsilon_{r s}^{-1}\left(\nabla \times W_{k} \hat{z}\right)\right]-k_{0}^{2} \mu_{r z} W_{j}^{*} W_{k}\right\},
$$

$$
T_{j, k}^{(1,2)}=-j k_{0} \int_{\partial \Gamma_{1,2}} d x W_{j}^{*} W_{k}
$$

In the implementation, the integration is calculated in the reference domain by the Gauss quadrature.

The relation between the surface magnetic field and surface magnetic current density is given by the surface integral equation 


$$
\begin{aligned}
H_{z}^{\mathrm{inc}}(x, y)= & \frac{1}{2} H_{z}(x, y)+j k_{0} \varepsilon_{r b} \int G_{p}^{(b)}\left[k_{b}\left(x-x^{\prime}\right), k_{b}(y\right. \\
& \left.\left.-y^{\prime}\right)\right] M_{z}\left(x^{\prime}\right) d x^{\prime}
\end{aligned}
$$

where $\varepsilon_{r b}$ and $k_{b}$ are the relative permittivity and wave number of the background material above (below) the upper (lower) boundary, with $b=1,2 ; H_{z}^{\text {inc }}$ is the incident field, and $G_{p}^{(b)}$ is the periodic Green's functions in these two regions. The periodic Green's functions contain the property of wave propagation in the upper and lower backgrounds. Note that another integral in the surface integral equation becomes zero, i.e.,

$$
\left.\int\left(\partial G_{p}^{(b)}\left[k_{b}\left(x-x^{\prime}\right), k_{b}\left(y-y^{\prime}\right)\right] / \partial y\right)\right|_{y=y^{\prime}} H_{z}\left(x^{\prime}\right) d x^{\prime}=0
$$

because

$$
\partial G_{p}^{(b)}\left[k_{b}\left(x-x^{\prime}\right), k_{b}\left(y-y^{\prime}\right)\right] / \partial y=0
$$

on the two boundaries where $y=y^{\prime}$. After transferring Eq. (8) into a weak form and expanding the magnetic field and magnetic current by the basis functions, the surface integral equation for the upper and lower boundaries can be written as two matrix equations, respectively,

$$
\begin{aligned}
\mathbf{U}^{(1)} \mathbf{H}^{b 1}+\mathbf{V}^{(1)} \mathbf{M}^{b 1} & =\mathbf{F}^{(1)}, \\
\mathbf{U}^{(2)} \mathbf{H}^{b 2}+\mathbf{V}^{(2)} \mathbf{M}^{b 2} & =\mathbf{F}^{(2)},
\end{aligned}
$$

where

$$
\begin{gathered}
U_{j, k}^{(1,2)}=\frac{1}{2} \int_{\partial \Gamma_{1,2}} d x W_{j}^{*} W_{k}, \\
V_{j, k}^{(1,2)}=j k_{0} \varepsilon_{r b} \int_{\partial \Gamma_{1,2}} \int_{\partial \Gamma_{1,2}} W_{j}^{*}(x) G_{p}^{(b)}\left[k_{b}(x\right. \\
\left.\left.-x^{\prime}\right), 0\right] W_{k}\left(x^{\prime}\right) d x d x^{\prime}, \\
F_{j}^{(1,2)}=\int_{\partial \Gamma_{1,2}} d x W_{j}^{*} H^{\text {inc }} .
\end{gathered}
$$

These integrals are calculated in the reference domain by the Gauss quadrature integration method. Note that the integral in Eq. (11) is a nonlocal integral, which means that the integral is nonzero even when the two basis functions in the integral do not overlap. The periodic Green's function has singular points at $x=x^{\prime}+m L_{x}$, with $m$ being any integer. In order to calculate the integral with high accuracy and efficiency, we use the Green's function in the wave number space. Grouping Eqs. (5) and (9), the scattering problem is described by the linear matrix equation problem. The total field in the calculation region, as well as transmission, reflection, and absorption coefficients, can be obtained by solving this matrix equation.

The periodic Green's function can be more easily evaluated in the wave number space than in the real space. In this paper, we restrict it to the case where the background is a homogeneous isotropic dielectric material, but the up- per and lower half spaces can be different. In a homogeneous space, the Green's function obeys the Helmholtz equation

$$
\nabla^{2} G^{b}\left(\mathbf{r}-\mathbf{r}^{\prime}\right)+k_{b}^{2} G^{b}\left(\mathbf{r}-\mathbf{r}^{\prime}\right)=-\delta\left(\mathbf{r}-\mathbf{r}^{\prime}\right),
$$

where $k_{b}$ is the wave vector in this homogeneous space. Making Fourier transform to the wave number space, solving the Green's function in the wave number space, and making inverse Fourier transform back to the real space, we have the Green's function in the homogeneous real space

$$
G^{b}\left(\mathbf{r}-\mathbf{r}^{\prime}\right)=\int \frac{e^{-j \mathbf{q} \cdot\left(\mathbf{r}-\mathbf{r}^{\prime}\right)}}{(2 \pi)^{2}\left(q^{2}-k_{b}^{2}\right)} d \mathbf{q}
$$

where $\mathbf{q}$ is the wave number vector. Since this is a $2 \mathrm{D}$ problem, the integral is in the $2 \mathrm{D}$ wave number space. If the system is periodic along the $\hat{x}$ direction, the periodic Green's function obeys the same Helmholtz equation with a source term $-\Sigma_{m} e^{-j k_{x} m L_{x}} \delta\left(\mathbf{r}-\mathbf{r}^{\prime}-m L_{x}\right)$, with $k_{x}$ being the Bloch wave number. As a result, the periodic Green's function can be written as

$$
\begin{aligned}
G_{p}^{b}\left(\mathbf{r}-\mathbf{r}^{\prime}\right) & =\sum_{m} e^{-j k_{x} m L_{x}} \int \frac{e^{-j \mathbf{q} \cdot\left(\mathbf{r}-\mathbf{r}^{\prime}-m L_{x} \hat{x}\right)}}{(2 \pi)^{2}\left(q^{2}-k_{b}^{2}\right)} d \mathbf{q} \\
& =\int \sum_{m} e^{-j k_{x} m L_{x}+j q_{x} m L_{x}} \frac{e^{-j \mathbf{q} \cdot\left(\mathbf{r}-\mathbf{r}^{\prime}\right)}}{(2 \pi)^{2}\left(q^{2}-k_{b}^{2}\right)} d \mathbf{q} .
\end{aligned}
$$

Making use of the Dirac comb function formula $\Sigma_{m} e^{-j\left(k_{x}-q_{x}\right) m L_{x}}=\left(2 \pi / L_{x}\right) \Sigma_{m} \delta\left(k_{x}-q_{x}-2 \pi m / L_{x}\right), \quad$ and performing the integration along $q_{x}$, we have

$$
G_{p}^{b}\left(\mathbf{r}-\mathbf{r}^{\prime}\right)=\sum_{m} e^{-j q_{x}^{m}\left(x-x^{\prime}\right)}\left(\frac{2 \pi}{L_{x}}\right) I_{m}\left(k_{b}, k_{x}\right),
$$

where $\quad q_{x}^{m}=k_{x}-2 \pi m / L_{x} \quad$ and $\quad I_{m}\left(k_{b}, k_{x}\right)$ $=\int e^{-j q_{y}\left(y-y^{\prime}\right)} d q_{y} /\left[(2 \pi)^{2}\left(q_{x}^{m}+q_{y}-k_{b}^{2}\right)\right]$. In our problem, on the surface of the computation domain, $y=y^{\prime}$, so the integration along the $q_{y}$ direction becomes $I_{m}\left(k_{b}, k_{x}\right)$ $=\left(2 \pi / L_{x}\right) \int d q_{y} /\left[(2 \pi)^{2}\left(q_{x}^{m}+q_{y}-k_{b}^{2}\right)\right]$, which can be evaluated by the method of contour integral. The integration result is $I_{m}\left(k_{b}, k_{x}\right)=\left(2 \pi / L_{x}\right)\left(1 / 4 \pi j \sqrt{k_{b}^{2}-q_{x}^{m}}\right)$. There are singular points when $q_{x}^{m}-k_{b}^{2}=0$, which defines the light cone. In the numerical implementation, we only need to calculate the problem away from the light cone most of the time. But if we need to calculate the result at the light cone, we could use another pair of parameters $\left(k_{b}, k_{x}\right)$ which is out of the light cone and very close to the original parameter to approximate. It is obvious that $I_{m}$ is a function of $k_{b}$ and $k_{x}$. Hence, the periodic Green's function is written as a summation of a set of plane waves, each of which is a separated function for $x$ and $x^{\prime}$. Inserting Eq. (16) into Eq. (11), the integral is written as 


$$
\begin{aligned}
V_{j, k}^{(1,2)}= & j k_{0} \varepsilon_{r b} \sum_{m=-N}^{N} I_{m}\left(k_{b}, k_{x}\right)\left[\int_{\partial \Gamma_{1,2}} e^{-j q_{x}^{m} x} W_{j}^{*}(x) d x\right] \\
& \times\left[\int_{\partial \Gamma_{1,2}} e^{j q_{x}^{m} x} W_{k}(x) d x\right],
\end{aligned}
$$

where $N$ is the cutoff of the summation, which is chosen to be the same as the number of nodal points at the surface so that the plane wave basis functions have approximately the same sampling density in terms of the number of points per wavelength. The two integrals in Eq. (17) can be calculated and stored in advance. Since the integrands are smooth functions, the integration results can be obtained very accurately by using the Gauss quadrature integration. The rapid calculation of the matrix elements $V_{j, k}^{(1,2)}$ can be implemented by the multiplication of the stored matrices of these integrals.

Some observable quantities, including the reflection rate, transmission rate, and absorption rate, are calculated from the SEM solution of the field pattern. The reflection rate and transmission rate are calculated by comparing the integral of the vertical component of Poynting vectors along the upper and lower boundaries with the incidence energy of the incident plane wave. The absorption rate can be calculated in two ways: one way is to use the energy conservation principle that gives the absorption rate as

$$
a=1-r-t,
$$

where $a$ is the absorption rate, $r$ is the reflection rate, and $t$ is the transmission rate; another way is to compare the dissipated energy

$$
P_{d}=\frac{1}{2} \int_{\Gamma} \omega \varepsilon^{\prime \prime}|\mathbf{E}|^{2} d \mathbf{r}
$$

with the incident energy, where $\varepsilon^{\prime \prime}$ is the imaginary part of the permittivity $\varepsilon$ and $\mathbf{E}$ is the electric field. In the systems that we have investigated, only gold has a nonzero imaginary part. The numerical results show that the two methods give the same absorption rate. As a result, all absorption is due to the loss in gold.

\section{NUMERICAL RESULTS}

We have first validated the method with a simple system having one half space filled with one material and the other half space filled with another material. As this scattering problem has an analytical solution, it is used to inspect the relative error of the SEM simulation results. Then, we use the method to calculate the scattering properties of a gold slit grid on a glass surface.

\section{A. Convergence of Relative Error}

The first example we calculate is scattering from a half space interface. The calculation region is divided into two rectangular elements, with the upper one being air and the lower one filled with a dielectric material with a permittivity equal to 3 as shown in Fig. 2(a). The result of the scattering under $45^{\circ}$ incidence is compared with the analytical result, and the relative errors of the field pattern
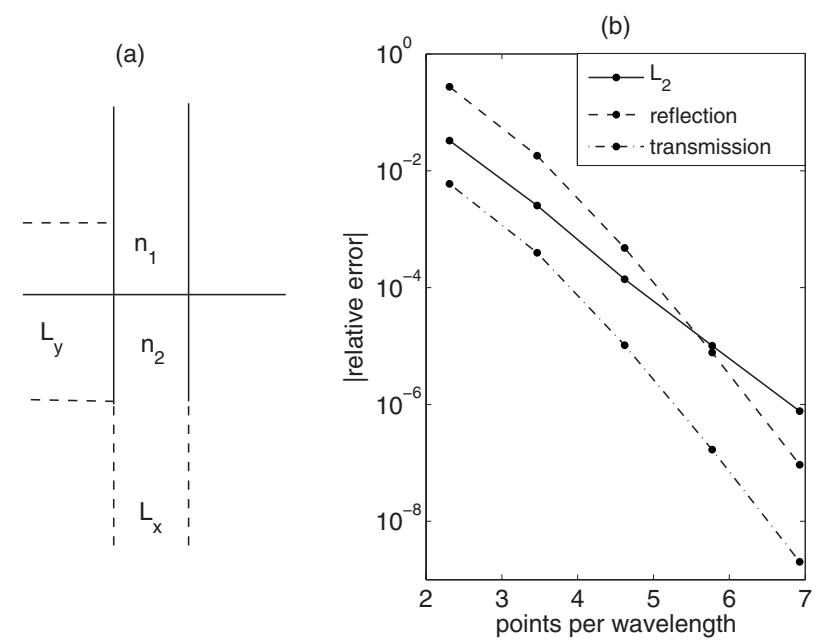

Fig. 2. (a) Geometry of a half space interface. (b) Relative errors of the field pattern, reflection rate, and transmission rate compared to the analytical solution of a half space interface versus the sampling density in terms of the number of points per wavelength.

( $L_{2}$ error), reflection rate, and transmission rate versus the sampling density in terms of the number of points per wavelength are plotted in Fig. 2(b). The $L_{2}$ relative error is defined as

$$
e=\frac{\sqrt{\int_{\Gamma}\left|H_{z}-H_{z}^{\text {exact }}\right|^{2} d \mathbf{r}}}{\sqrt{\int_{\Gamma}\left|H_{z}^{\text {exact }}\right|^{2} d \mathbf{r}}}
$$

where $H_{z}^{\text {exact }}$ is the exact field pattern of the analytical solution. As we can observe, the calculation result with a sampling density fewer than five points per wavelength can have a relative error less than $10^{-3}$. The straight lines in Fig. 2(b) show that the SEM has an exponential convergence.

\section{B. Extraordinary Transmission and Absorption of a Gold Slit Grid}

The material configuration of the system being investigated in this subsection is shown in Fig. 3. Since the system is periodic along the $\hat{x}$ direction, only one unit cell with width $L_{x}$ is plotted. Figure 3 (a) shows a square gold grid. A gold film with thickness $d$ is deposited between two kinds of dielectric materials filled in the upper and lower backgrounds. A grid of slits in the gold film with width $s$ is filled with the same dielectric material as the lower background. In applications, the upper background could be a prism and the lower background could be a liquid. The fill factor is defined as $f=s / L_{x}$. A plane wave is incident from the upper space. The permittivity of gold is frequency dependent and its dispersion curve is described in [16-18]. In this example, the relative permittivity values of the upper and lower background materials are $\varepsilon_{r 1}$ $=1.723^{2}$ and $\varepsilon_{r 1}=1.333^{2}$, respectively. Figure $3(\mathrm{~b})$ shows a triangular gold grid, which has the same area of gold filled in one unit cell as the system in Fig. 3(a).

The transmission (a) and absorption (b) rates versus wavelength and incidence angle of a square gold grid sys- 
(a)
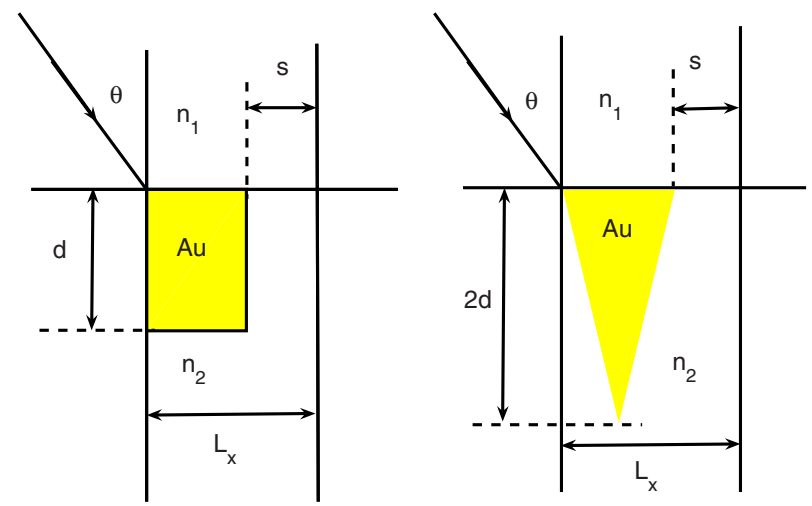

Fig. 3. (Color online) Two configurations of a unit cell of a gold slit grid. The upper and lower half spaces are filled with dielectric materials with reflective indices $n_{1}$ and $n_{2}$, respectively. The third material, gold in this case, is deposited under the interface of the two dielectric materials. In system (a), the gold grid is rectangular; the period along the $\hat{x}$ direction is $L_{x}$, the thickness of the gold film is $d$, and the width of the slit is $s$. The fill factor is defined as $f=s / L_{x}$ and $\theta$ is the angle of incidence of the plane wave. In the system (b), the gold grid is triangular; the period and width of the slit are the same as in system (a), but the height of the gold triangle is $2 d$, so that the area of gold is the same as in (a).

tem with $L_{x}=200 \mathrm{~nm}, d=200 \mathrm{~nm}$, and $f=0.2$ are plotted in Fig. 4. At a wavelength around $1000 \mathrm{~nm}$, the transmission rate is very large when the incidence angle is less than $50.7^{\circ}$, which is the critical angle of the total internal reflection. The absorption rate is very large in several regions. The large absorption below $500 \mathrm{~nm}$ is due to the relatively large imaginary part of the electric permittivity and the enhanced energy confinement inside the metal due to the close to zero real part of the electric permittivity. The other regions are some small area regions at the wavelength around $700 \mathrm{~nm}$ and incidence angle larger than $50.7^{\circ}$. In these regions, the imaginary part of the permittivity of gold is very small; therefore, the extraordinary absorptions are due to the coupling to the SPP modes in the subwavelength metal structure. The inci- (a)

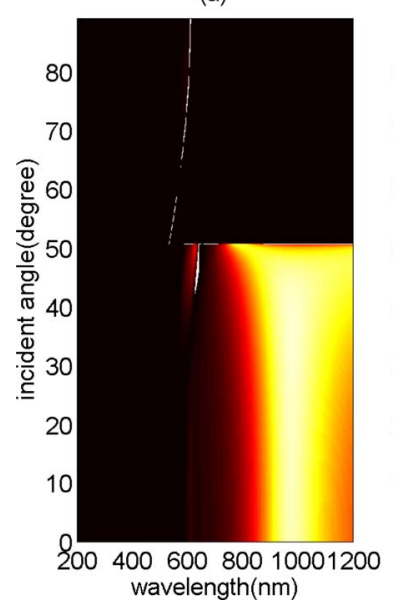

(b)

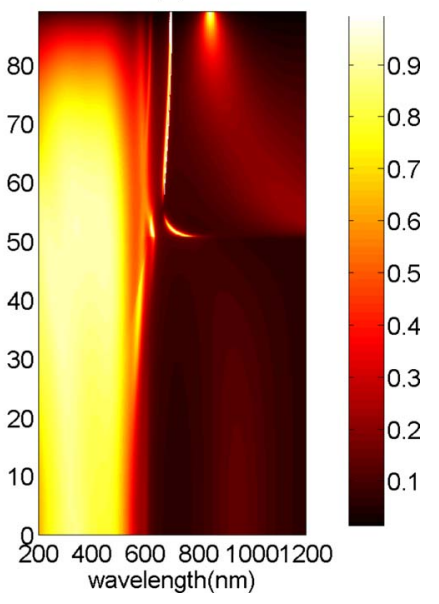

Fig. 4. (Color online) (a) Transmission rate and (b) absorption rate of a square gold grid with $L_{x}=200 \mathrm{~nm}, d=200 \mathrm{~nm}$, and $f$ $=0.2$.

dent light is coupled to the SPP modes, which focus the field in the gold film and promote the absorption, and eventually the energy is damped into the metal.

As a comparison, we calculated another square gold grid system, which has a thickness $d=50 \mathrm{~nm}$ while the other parameters remain the same as the previous system. The transmission (a) and absorption (b) rates are plotted in Fig. 5. The region with a large transmission rate shifts up to a larger wavelength. The absorption rate in the large region with a wavelength under $500 \mathrm{~nm}$ becomes smaller, which proves that the large absorption rate in this region is attributed to the internal absorption of gold depending on the thickness of the gold film. The absorption rate at the other small region is still large. One more small region with a large absorption rate appears at the wavelength about $600 \mathrm{~nm}$ and incidence angle larger than $50.7^{\circ}$. As a result, the large absorption rate at these small regions is proved to be the extraordinary absorption because of the structure of the system. As a comparison, the numerical results given by the Fourier modal method (FMM) [19-23] and by the SEM are plotted together in Figs. 6(a) and 6(b). The agreement is very good. We have also compared the numerical results of a system with a fill factor $f=0$ with analytical solutions in Figs. 6(c) and 6(d). The straight line in Fig. 6(d) shows that the numerical SEM solution has an exponential convergence and even the second order SEM has a relative error smaller than $0.1 \%$. Another advantage of the SEM is that it can solve systems with more complicated spatial structures by making the mesh conformal to the geometric structure. The transmission (a) and absorption (b) rates of a triangular gold grid with the same area of gold filled in one unit cell as the square gold grid in Fig. 5 are plotted in Fig. 7. It is shown that the large region with a high absorption rate is almost the same, which is only decided by the area of gold filled. The small regions with a high absorption rate become wider and have different locations; the region with a high transmission rate also becomes larger. This is because the triangular gold grid has a different dispersive relation of the SPP modes from the square gold grid.

(a)

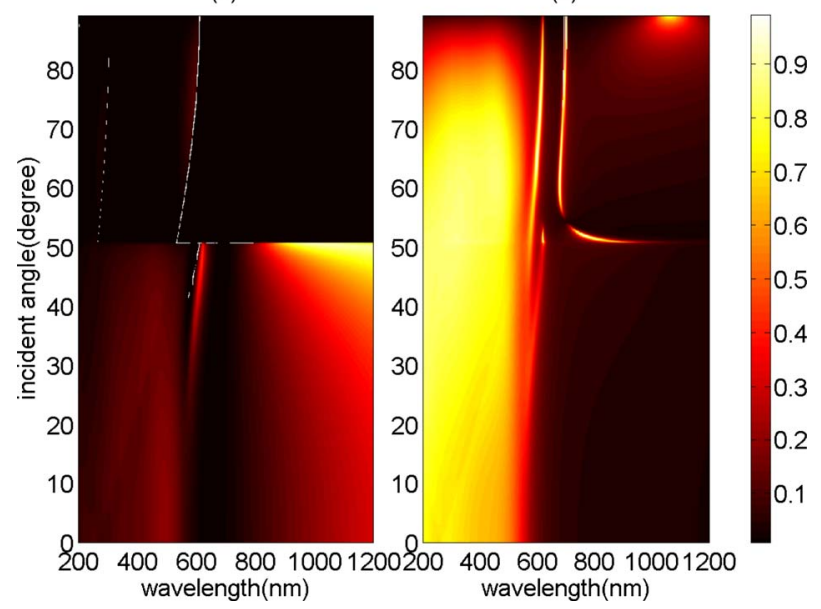

Fig. 5. (Color online) (a) Transmission rate and (b) absorption rate of a square gold grid with $L_{x}=200 \mathrm{~nm}, d=50 \mathrm{~nm}$, and $f$ $=0.2$. 
(a)

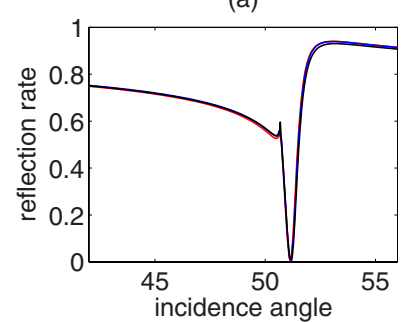

(c)

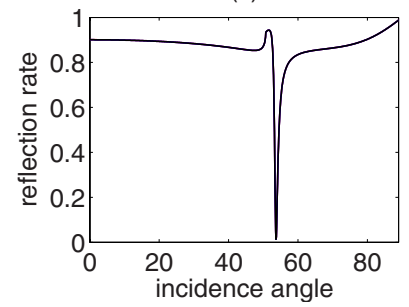

(b)

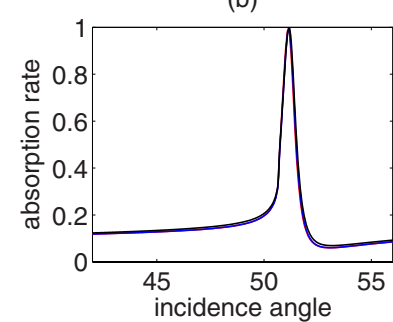

(d)

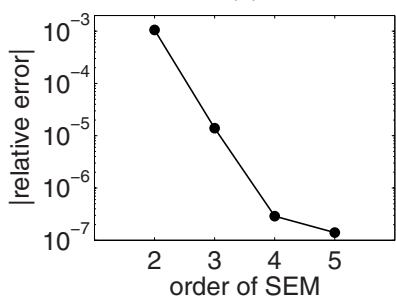

Fig. 6. (Color online) Accuracy analysis of the SEM. Comparison of the reflection rate (a) and absorption rate (b) obtained by the fifth order SEM (blue dotted line), the ninth order SEM (red dashed line), and the FMM (black line) for the system in Fig. 5. (c) Comparison of the reflection rate obtained by the third order SEM (blue dotted line), the fifth order SEM (red dashed line), and the analytical solution (black line) with fill factor $f=0$ and $200 \mathrm{~nm}$ thick gold film. (d) The relative error of reflection rate versus the order of the SEM. The wavelength of these calculations is $850 \mathrm{~nm}$.

For both systems in Figs. 4, 5, and 7, there is a small transmission rate at a wavelength under $600 \mathrm{~nm}$ and incidence angle larger than $50.7^{\circ}$. A third square gold grid system with $L_{x}=600 \mathrm{~nm}, d=600 \mathrm{~nm}$, and $f=0.2$ was simulated as a comparison. The transmission rate is plotted in Fig. 8, which shows that there is a large region with a large transmission rate at the wavelength under $2000 \mathrm{~nm}$ and incidence angle larger than $50.7^{\circ}$. This transmission at the angle larger than the critical angle is attributed to the assistance of periodic structure. Considering the system without the gold grid, the relation between incident angle $\theta_{\text {in }}$ and refractive angle $\theta_{\text {re }}$ is $\sin \left(\theta_{\text {in }}\right) n_{1} k_{0}$ $=\sin \left(\theta_{\mathrm{re}}\right) n_{2} k_{0}=k_{x}$, with $k_{x}$ being the wave number of the traveling wave along the $\hat{x}$ direction, so the phase along

(a)

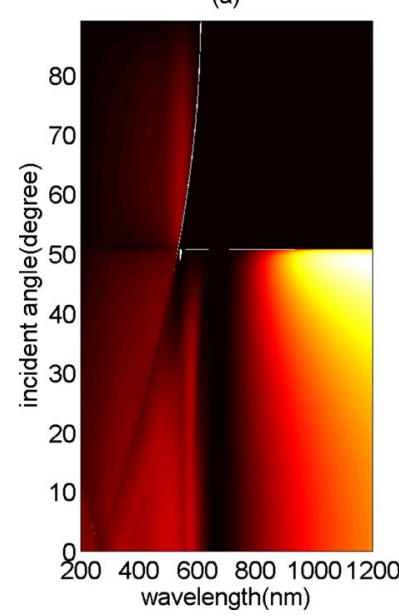

(b)

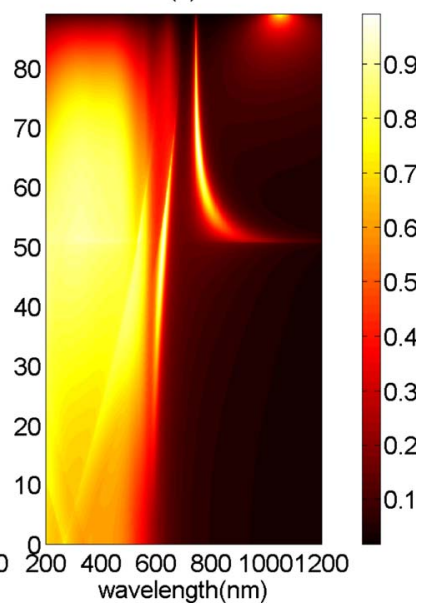

Fig. 7. (Color online) (a) Transmission rate and (b) absorption rate of a triangular gold grid with $L_{x}=200 \mathrm{~nm}, 2 d=100 \mathrm{~nm}$, and $f=0.2$.

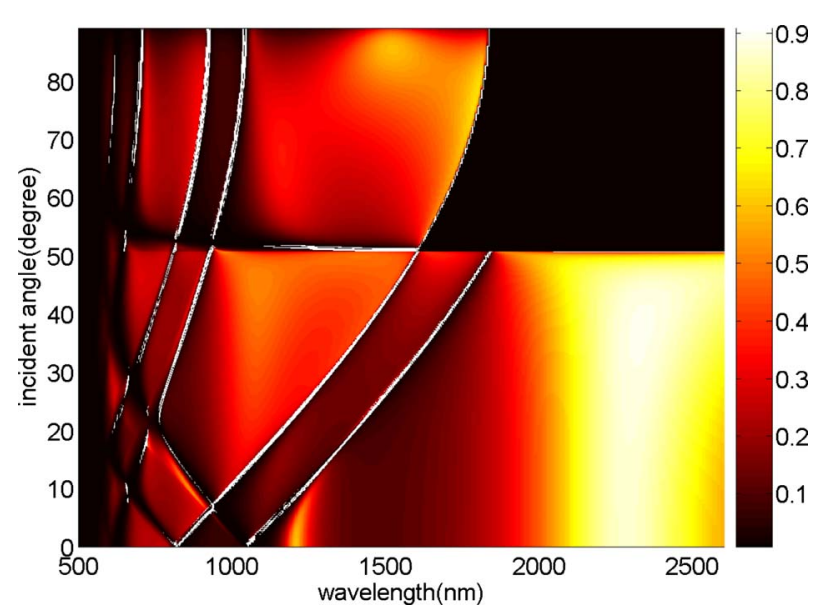

Fig. 8. (Color online) Transmission rate of a square gold grid with $L_{x}=600 \mathrm{~nm}, d=600 \mathrm{~nm}$, and $f=0.2$.

the $\hat{x}$ direction matches at the interface. If $k_{x}$ is larger than $n_{2} k_{0}$, then $k_{y}$ in the lower half space must be imaginary, because $\left(n_{2} k_{0}\right)^{2}=k_{x}^{2}+k_{y}^{2}$. In this case, the refractive wave becomes an evanescent wave that does not transfer energy along the $\hat{y}$ direction, so the incident wave is totally reflected. When there is a periodic grid with period $L_{x}$, the wave number along the $\hat{x}$ direction of the refracted wave could be $k_{x}+2 \pi m / L_{x}$, with $m$ being any integer to satisfy the phase matching condition. When one of these wave numbers is smaller than $n_{2} k_{0}$, the corresponding $k_{y}$ in the lower half space is real, so the refracted wave is a traveling wave along the $\hat{y}$ direction and the transmission rate is nonzero. Obviously, there is a possibility of the value of $m$ being -1 or a smaller integer. When $L_{x}$ is larger, $2 \pi / L_{x}$ becomes smaller, so $m$ with a larger absolute value can satisfy the traveling wave condition. As a result, the transmission at the incidence angle larger than $50.7^{\circ}$ is larger when $L_{x}=600 \mathrm{~nm}$ than that when $L_{x}=200 \mathrm{~nm}$. Note that when the grid consists of a dielectric material with a positive permittivity value, there is also transmission at this condition. This means that this transmission is not attributed to the negative permittivity of gold.

\section{CONCLUSION}

The spectral element method (SEM) together with the method of moments (MoM) is used to simulate the 2D scattering of a gold slit grid with a 1D periodic structure. Since the SEM has very high accuracy and efficiency, the MoM part has been improved so that the whole simulation can have high accuracy and efficiency. As a result, the periodic Green's function is transformed into the wave number representation given by Eq. (16). The calculation of the matrix elements in the MoM using this form of periodic Green's function is easier to achieve high accuracy. The solution convergence is tested by comparing the calculation results of the scattering of a half space interface with analytical solutions. The numerical results show an exponential convergence. The method is then used to investigate the scattering properties of gold slit grids. The extraordinary transmission and extraordinary absorption are investigated over the incidence angle-wavelength pa- 
rameter space. An extraordinary transmission rate at some wavelength and extra-large absorption at some small regions of the wavelength-incidence angle parameter space are found. The extraordinary absorption at some regions of the wavelength-incidence angle parameter space is due to the excitation of the SPP modes in the subwavelength metal structures. The transmission at an incidence angle larger than the critical angle of the total internal reflection is enhanced by the periodic grid.

\section{ACKNOWLEDGMENT}

The support from the National Science Foundation (NSF) under grant CCF-0621862 is appreciated.

\section{REFERENCES}

1. J.-H. Lee and Q. H. Liu, "An efficient 3-D spectral element method for Schrodinger equation in nanodevice simulation," IEEE Trans. Comput.-Aided Des. 24, 1848-1858 (2005).

2. G. C. Cohen, Higher-Order Numerical Methods for Transient Wave Equations (Springer, 2001).

3. J.-H. Lee, T. Xiao, and Q. H. Liu, "A 3-D spectral element method using mixed-order curl conforming vector basis functions for electromagnetic fields," IEEE Trans. Microwave Theory Tech. 54, 437-444 (2006).

4. M. Luo, Q. H. Liu, and Z. Li, "Spectral element method for band structures of two-dimensional anisotropic photonic crystals,” Phys. Rev. E 79, 026705 (2009).

5. J. Liu and Q. H. Liu, "A novel radiation boundary condition for finite-element method," Microwave Opt. Technol. Lett. 49, 1995-2002 (2007).

6. F. Q. Hu, "A spectral boundary integral equation method for the 2D Helmholtz equation," J. Comput. Phys. 120, 340-347 (1995).

7. J. Liu and Q. H. Liu, "A spectral integral method (SIM) for periodic and nonperiodic structures," IEEE Microw. Wirel. Compon. Lett. 14, 97-99 (2004).

8. H. Raether, Surface Plasmons on Smooth and Rough Surfaces and on Gratings (Springer-Verlag, 1988).

9. E. Kretschmann and H. Raether, "Radiative decay of nonradiative surface plasmons excited by light," Z. Naturforsch. A 23, 2135-2136 (1968).

10. A. Otto, "Excitation of nonradiative surface plasma waves in silver by the method of frustrated total reflection," Z. Phys. 216, 398-410 (1968).

11. Y.-Y. Teng and E. A. Stern, "Plasma radiation from metal grating surfaces," Phys. Rev. Lett. 19, 511-514 (1967).

12. R. H. Ritchie, E. T. Arakawa, J. J. Cowan, and R. N. Hamm, "Surface-plasmon resonance effect in grating diffraction," Phys. Rev. Lett. 21, 1530-1533 (1968).

13. C. J. Alleyne, A. G. Kirk, R. C. McPhedran, N.-A. P. Nicorovici, and D. Maystre, "Enhanced SPR sensitivity using periodic metallic structures," Opt. Express 15, 8163-8169 (2007).

14. K. M. Byun, S. J. Kim, and D. Kim, "Design study of highly sensitive nanowire-enhanced surface plasmon resonance biosensors using rigorous coupled wave analysis," Opt. Express 13, 3737-3742 (2005).

15. K. M. Byun, M. L. Shuler, S. J. Kim, S. J. Yoon, and D. Kim, "Sensitivity enhancement of surface plasmon resonance imaging using periodic metallic nanowires," J. Lightwave Technol. 26, 1472-1478 (2008).

16. P. G. Etchegoin, E. C. Le Ru, and M. Meyer, "An analytic model for the optical properties of gold," J. Chem. Phys. 125, 164705 (2006).

17. P. G. Etchegoin, E. C. Le Ru, and M. Meyer, "Erratum: An analytic model for the optical properties of gold," J. Chem. Phys. 127, 189901(E) (2007).

18. A. D. Rakic, A. B. Djurisic, J. M. Elazar, and M. L. Majewski, "Optical properties of metallic films for verticalcavity optoelectronic devices," Appl. Opt. 37, 5271-5283 (1998).

19. H.-S. Leong, J. Guo, R. G. Lindquist, and Q. H. Liu, "Surface plasmon resonance in nanostructured metal films under the Kretschmann configuration” J. Appl. Phys. 106, 124314 (2009).

20. T. Tamir, H. C. Wang, and A. A. Oliner, "Wave propagation in sinusoidally stratified dielectric media," IEEE Trans. Microwave Theory Tech. 12, 323-335 (1964).

21. C. B. Burckhardt, "Diffraction of a plane wave at a sinusoidally stratified dielectric grating," J. Opt. Soc. Am. 56, 1502-1508 (1966).

22. M. G. Moharam and T. K. Gaylord, "Rigorous coupled-wave analysis of metallic surface-relief gratings," J. Opt. Soc. Am. A 3, 1780-1787 (1986).

23. M. G. Moharam, E. B. Grann, D. A. Pommet, and T. K. Gaylord,"Formulation for stable and efficient implementation of the rigorous coupled-wave analysis of binary gratings,” J. Opt. Soc. Am. A Opt. Image Sci. Vis 12, 1068-1076 (1995). 